\title{
Real-Time, Video-Rate and Depth-Resolved Imaging of Radio-Frequency Ablation using All-Optical Ultrasound
}

\author{
Erwin J Alles ${ }^{*, \dagger}$, Richard J Colchester*,†, Yousuf Makki*, Sacha Noimark ${ }^{*}, \dagger, \ddagger$, \\ Edward Z Zhang*, Paul C Beard*, ${ }^{*}$, Malcolm C Finlay*, ${ }^{*}$, and Adrien E Desjardins ${ }^{*}, \dagger$ \\ *Department of Medical Physics \& Biomedical Engineering, University College London, London, United Kingdom \\ ${ }^{\dagger}$ Wellcome / EPSRC Centre for Surgical and Interventional Sciences, University College London, London, United Kingdom \\ ${ }_{\ddagger}^{\ddagger}$ Materials Chemistry Research Centre, Department of Chemistry, University College London, London, United Kingdom \\ $\S$ William Harvey Cardiovascular Research Institute, Queen Mary University of London and Barts Heart Centre, United Kingdom \\ E-mail: e.alles@ucl.ac.uk
}

\begin{abstract}
Radio-frequency (RF) ablation is an effective minimally invasive treatment for atrial fibrillation, where RF fields are delivered by a catheter within the heart. In current clinical practice, this procedure is performed without real-time feedback about the viability of tissue beneath the ablation lesion. As a result, it can be challenging to perform complete ablation of the target tissue whilst avoiding damage to adjacent structures. Recently, all-optical ultrasound imaging, where ultrasound is both generated and detected using light, has been demonstrated as a viable imaging modality exhibiting high image resolution, strong miniaturisation potential, and resilience to interfererence by electromagnetic (EM) fields from the RF ablation catheter. In this work, the suitability of all-optical ultrasound to monitoring RF ablation lesion delivery is investigated using a bench-top imaging system. This system is capable of real-time, video-rate $2 D$ imaging at an image depth of $15 \mathrm{~mm}$, a resolution of $100 \mu \mathrm{m}$, a dynamic range of $35 \mathrm{~dB}$, and a sustained frame rate of $9 \mathrm{~Hz}$. In this paper we present the first real-time, video-rate all-optical ultrasound images acquired during RF ablation, and demonstrate how the imaging system was able to clearly visualise the lesion growth at a contrast of up to $30 \mathrm{~dB}$. In addition, we demonstrated how lesion growth in inhomogeneous tissue can progress in an erratic fashion. High-quality imaging during treatment will enable optimisation of the ablation parameters during treatment, and will minimise both treatment times and complication rates.

Index Terms-All-optical ultrasound, radio-frequency ablation, ablation lesion monitoring
\end{abstract}

\section{INTRODUCTION}

Atrial fibrillation is a heart condition affecting an estimated $1 \%$ of the adult population [1]. With this condition, specific regions of the heart no longer contract synchronously, which results in an irregular heart rate and elevated risk of stroke [2]. To restore full synchronicity, radio-frequency (RF) ablation of heart tissue is widely used as a treatment when pharmacological treatment is inadequate. With this procedure,

This work was supported by an ERC Starting Grant [310970 MOPHIM], an ERC Advanced Grant [741149], the EU project FAMOS [FP7 ICT, Contract 317744], the Innovative Engineering for Health award by the Wellcome Trust [WT101957] and the EPSRC [NS/A000027/1], the EPSRC Healthcare Technologies Challenge Award [EP/N021177/1], and the EPSRC Doctoral Training Partnership [EP/N509577/1]. a catheter tip emitting intense electromagnetic (EM) RF fields is placed against cardiac tissue, and tissue ablation is induced by absorption of these fields.

Radio-frequency ablation catheters are typically positioned within the heart under guidance of X-ray fluoroscopy. X-ray fluoroscopy offers good catheter visibility, thus facilitating its positioning, but its soft-tissue contrast is insufficient to visualise heart tissue and ablation lesion progression. As a result, clinicians purely rely on initial positioning and tactile feedback from the catheter to ensure tissue contact and catheter positioning. However, the lack of real-time treatment lesion monitoring introduces the risks of catheter motion, loss of tissue contact, or over-treatment, which can have life-threatening consequences.

Several interventional imaging modalities have been demonstrated to achieve high-resolution, real-time RF ablation lesion visualisation using a minimally invasive device. For instance, M-mode imaging was demonstrated with optical coherence tomography catheters [3], through optical spectroscopy [4], and using ultrasound catheters comprising electronic transducers [5], [6]. In addition, real-time photoacoustic imaging has been demonstrated in vitro using either fully external imaging probes [7], [8] or by combining interstitial light delivery with external ultrasound detectors [9]. However, real-time 2D or 3D ultrasound visualisation of lesion formation from inside the heart has remained elusive.

Recent advances in all-optical ultrasound imaging, where ultrasound is both generated and detected using light, have resulted in miniature fibre-optic imaging probes [10]-[13] that are inherently immune to EM interference. They are ideally suited to interventional imaging applications, and here we consider their application to RF ablation lesion monitoring. We present the first real-time, video-rate 2D all-optical ultrasound imaging of RF tissue ablation. Using a bench-top imaging setup [14], we monitored RF ablation lesion formation at a contrast of up to $30 \mathrm{~dB}$, and investigated the growth pattern of RF ablation lesions for different tissue types. 

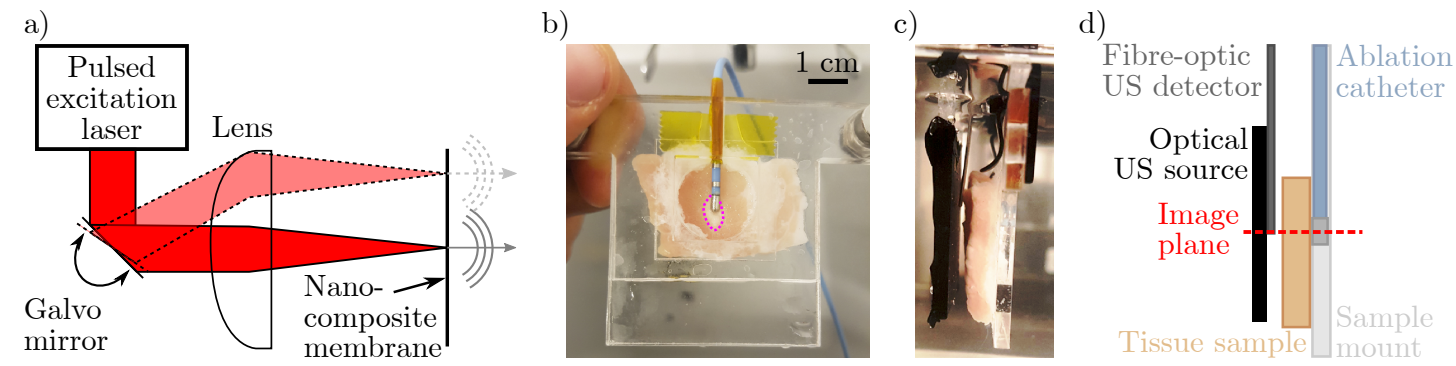

Fig. 1. The all-optical ultrasound imaging paradigm and experimental setup. (a) Schematic of the all-optical ultrasound imaging paradigm. Eccentrically focussed, pulsed excitation light was delivered to a nanocomposite optical ultrasound generating membrane, where it was converted into ultrasound via the photoacoustic effect. Using a set of galvo mirrors, the resulting optical ultrasound source was scanned across an aperture to synthesise an imaging array. Pulse-echo signals were recorded using a single fibre-optic ultrasound detector (not shown). (b) Photograph of the custom sample mount used to keep the ablation catheter in direct contact with the tissue. The white region observed around the catheter tip (annotated in purple) corresponds to the ablation lesion. (c) Photograph and (d) schematic of the sample mount placed in the all-optical ultrasound imaging setup.

\section{Methods}

\section{A. Optical ultrasound generation}

Ultrasound was generated optically by delivering focussed, pulsed excitation light (wavelength: $1064 \mathrm{~nm}$, pulse energy: $76 \mu \mathrm{m}$, pulse duration: $5 \mathrm{~ns}$, repetition rate: $3 \mathrm{kHz}$; FQS400-1-Y-1064, Elforlight, U.K.) to an optically absorbing membrane, where it was converted into ultrasound via the photoacoustic effect [15]. This membrane was fabricated from a nanocomposite of multi-walled carbon-nanotubes and polydimethylsiloxane (PDMS) to generated high pressures (MPa range) and bandwidths $(25-30 \mathrm{MHz})$ [16]-[18]. Using fast scanning optics, a single optical ultrasound source was rapidly steered across this membrane to synthesise a linear imaging aperture, thus enabling real-time and video-rate $2 \mathrm{D}$ imaging (sustained frame rate: $9 \mathrm{~Hz}$, imaging depth: $15 \mathrm{~mm}$, resolution: $100 \mu \mathrm{m})$. The source array comprised 200 sources distributed along a linear aperture (width: $13 \mathrm{~mm}$ ) using a trigonometric asin pattern [14]. A schematic of the imaging paradigm is shown in Fig. 1(a).

\section{B. Optical ultrasound detection}

Back-scattered ultrasound waves were recorded using a fibreoptic ultrasound detector comprising a Fabry-Pérot cavity [19] (noise-equivalent pressure: $40 \mathrm{~Pa}$, bandwidth: up to $80 \mathrm{MHz}$, omnidirectional response). Pressure variations of the impinging ultrasound waves resulted in changes in reflectivity of this cavity [20], [21], which were detected using a photodiode (DET01CFC, Thorlabs, Germany), amplified (+30 dB; DHPVA-200, Femto, Germany), and digitised (125 MSa/s, 14 bit; M4i.4420-x8, Spectrum, Germany) without signal averaging. The acquired data were reconstructed into B-mode images using the delay-and-sum algorithm [22], and displayed (after envelope detection) on a logarithmic scale spanning a dynamic range of $35 \mathrm{~dB}$.

\section{Radio-frequency ablation and tissue preparation}

A passively cooled, monopolar ablation catheter (max power: $30 \mathrm{~W}$, max temperature: $65^{\circ} \mathrm{C}$, treatment duration: $60 \mathrm{~s}$; IBI1500T, Irvine Biomedical, CA, USA) was used to generate RF ablation lesions. A custom mounting frame facilitated tissue mounting and ensured reliable contact between the catheter tip and the tissue sample, and is shown in Fig. 1(b-d). The ablation catheter was positioned behind the tissue sample to avoid acoustic shadowing from the metal catheter tip. Both structurally homogeneous (fresh organic chicken breast) and inhomogeneous (fresh organic pork belly) tissue samples were used to investigate how variations in tissue structure affect RF lesion formation. Thin tissue samples were manually cut (thickness: $4-10 \mathrm{~mm}$ ) and bonded to the sample mount using cyanoacrylate.

\section{RESULTS}

Real-time, video-rate imaging of a homogeneous tissue sample undergoing RF ablation revealed that the ablation lesion formation could clearly be visualised at a contrast of up to $30 \mathrm{~dB}$ (Fig. 2; the full video is available from youtu.be/HR5HFuOejE). The lesion was observed to exhibit isotropic growth away from the catheter tip, and the lesion size determined from all-optical ultrasound images $(6 \mathrm{~mm} \times 4 \mathrm{~mm}$ post-ablation) was identical to that determined from section photographs.

When an inhomogeneous tissue sample was imaged during RF ablation, the lesion again was clearly visible (Fig. 3(c)). However, the lesion growth pattern was much more erratic than that observed in the homogeneous tissue sample (as can be appreciated from the corresponding video available at youtu.be/6ZD7cEj4AYs), exhibiting "tendril-like" growth along preferred directions. Good correspondence was observed between the lesion shape judged from all-optical ultrasound images and the shape, size and orientation of the features (e.g., fat striations) visible in the corresponding tissue section (Fig. 3(a)). In this photograph, the area around the striation appears less treated than the surroundings, as was suggested by the lesion appearance in the all-optical ultrasound images.

In addition, an increase with time in the overall brightness of the all-optical ultrasound images was observed. This could be attributed to the strong temperature dependency of the speed of sound in fat; as the pork belly tissue (containing a high concentration of fat) increased in temperature, the acoustic contrast between fat and water increased. The ablation catheter 
a)

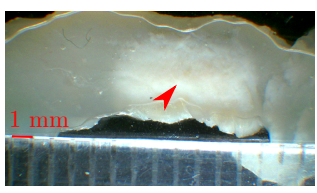

b)

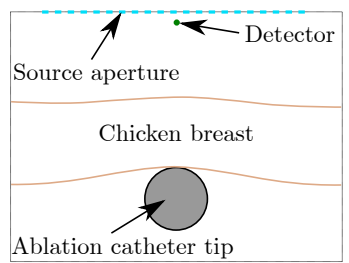

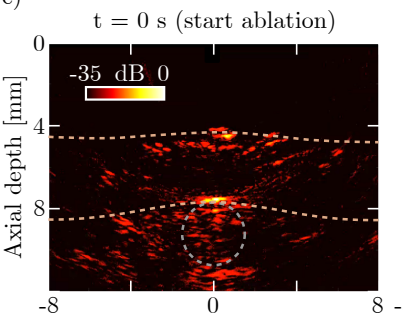
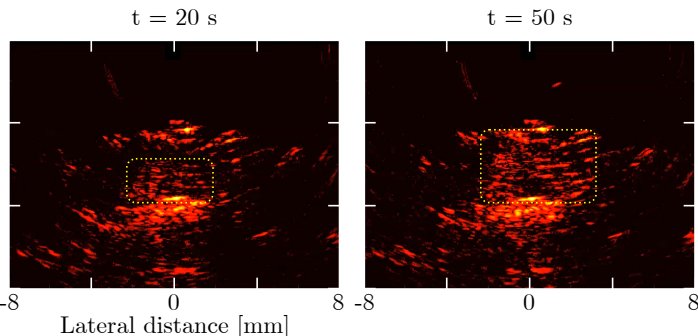

Fig. 2. All-optical ultrasound imaging of homogeneous tissue. (a) Photograph of the homogeneous tissue sample (chicken breast) post-ablation $(t>60 \mathrm{~s})$, showing a cross-section coinciding with the $2 \mathrm{D}$ all-optical ultrasound image plane. The ablation lesion (the white, "cloudy" region indicated by the red arrow head) is clearly visible. (b) Schematic of the image plane (ablation lesion not shown). A section of chicken breast was positioned a distance of 4 mm away from the imaging aperture (dashed line), and placed in direct contact with the tip of the ablation catheter that was positioned behind the tissue. A single fibre-optic detector was placed directly within the image plane, centered laterally, and axially offset by 1.0 mm. (c) All-optical ultrasound images of the tissue sample at different time points during the treatment. Time $t=0$ corresponds to the start of the $60 \mathrm{~s}-$ long ablation. The dashed curves (left panel) show the location of the tissue and catheter tip; the yellow dotted boxes (middle and right panels) the approximate location and size of the ablation lesion. The full video of this experiment is available on youtu.be/H-R5HFuOejE.

tip was not visualised as the strong acoustic inhomogeneities throughout the sample inhibited accurate image reconstruction at large depths.

\section{Discussion AND CONCLUSION}

In this work, we applied real-time, video-rate all-optical ultrasound imaging to for the first time enable RF ablation lesion visualisation during treatment. To this aim, a bench-top alloptical ultrasound imaging setup was used that generated 2D images at an image depth of $15 \mathrm{~mm}$, a resolution of $100 \mu \mathrm{m}$, a dynamic range of $35 \mathrm{~dB}$, and a sustained frame rate of $9 \mathrm{~Hz}$. Ablation lesions were clearly visualised, despite the strong EM fields generated by the ablation catheter and the corresponding sharp temperature gradients.

Real-time imaging during RF ablation was performed using both homogeneous and inhomogeneous tissue samples, and different lesion growth patterns were observed for these tissues. The observation of erratic lesion growth for inhomogeneous tissue highlights the need for true 2D or 3D imaging: if isotropic lesion growth was assumed, or lesion progression was extrapolated from single-channel M-mode measurements, the estimated shape and size of the lesion could have differed significantly, thus risking over- or under-treatment.

While the cardiac tissue targeted during RF ablation treatment of atrial fibrillation can be largely homogeneous, tissues encountered in other clinical contexts, such as liver tumours in interventional oncology, can be highly inhomogeneous. Greater levels of inhomogeneity may heighten our uncertainties about how RF ablation lesions progress. Additional uncertainty can be introduced by cooling effects due to local blood flow [23], [24]. In future studies we will therefore investigate the lesion growth in a range of tissue types and blood flow conditions. Detailed comparisons to histological analyses will be valuable to better understand how the alloptical ultrasound images relate to tissue changes induced by RF ablation.

The lesion size and shape have in this work been determined offline through visual analysis of the recorded videos. In future work, we will explore the use of statistical parameters derived from data along spatial and temporal dimensions. Ultimately, these could result in real-time and fully automatic RF ablation lesion contouring to facilitate image interpretation in a clinical setting, and provide clinicians with invaluable feedback during treatment.

The presented work was performed using a bench-top imaging system that is, at present, not suitable for interventional applications due to size constraints. However, by leveraging previous advances in sub-millimetre fibre-optic all-optical ultrasound imaging probes, all-optical ultrasound imaging could be integrated with an ablation catheter. Such an integrated device will enable minimally invasive ablation treatment under continuous guidance and monitoring, and will allow for optimisation and verfication of the treatment parameters in order to reduce prodecure times and complication rates.

\section{REFERENCES}

[1] V Malladi, PS Naeini, M Razavi, CD Collard, JM Anton, and DA Tolpin Endovascular ablation of atrial fibrillation. Anesthesiology: The Journal of the American Society of Anesthesiologists, 120(6):1513-1519, 2014.

[2] NHS. Atrial fibrillation https://www.nhs.uk/conditions/atrial-fibrillation/, Accessed 16 August 2018.

[3] BJ Vakoc, GJ Tearney, and BE Bouma. Real-time microscopic visualization of tissue response to laser thermal therapy. Journal of Biomedical Optics, 12(2):020501, 2007.

[4] RP Singh-Moon, CC Marboe, and CP Hendon. Near-infrared spectroscopy integrated catheter for characterization of myocardial tissues: preliminary demonstrations to radiofrequency ablation therapy for atrial fibrillation. Biomedical optics express, 6(7):2494-2511, 2015.

[5] M Wright, E Harks, S Deladi, F Suijver, M Barley, A van Dusschoten, S Fokkenrood, F Zuo, F Sacher, and M Hocini. Real-time lesion assessment using a novel combined ultrasound and radiofrequency ablation catheter. Heart Rhythm, 8(2):304-312, 2011.

[6] DE Haines, M Wright, E Harks, S Deladi, S Fokkenrood, R Brink, $\mathrm{H}$ Belt, AF Kolen, N Mihajlovic, and F Zuo. Near-field ultrasound imaging during radiofrequency catheter ablation: tissue thickness and epicardial wall visualization and assessment of radiofrequency ablation lesion formation and depth. Circulation: Arrhythmia and Electrophysiology, 10(12):e005295, 2017.

[7] N Dana, L Di Biase, A Natale, S Emelianov, and R Bouchard. In vitro photoacoustic visualization of myocardial ablation lesions. Heart Rhythm, 11(1):150-157, 2014.

[8] S Iskander-Rizk, P Kruizinga, AF van der Steen, and G van Soest. Spectroscopic photoacoustic imaging of radiofrequency ablation in the left atrium. Biomedical optics express, 9(3):1309-1322, 2018. 
a)

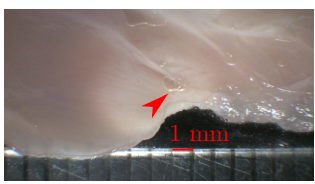

b)

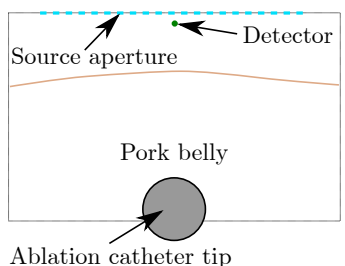

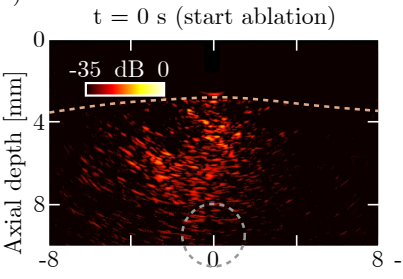

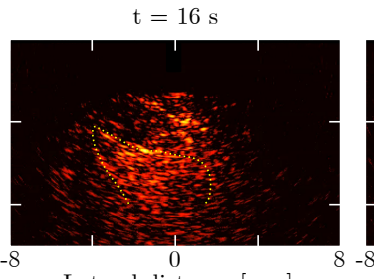

Lateral distance $[\mathrm{mm}]$

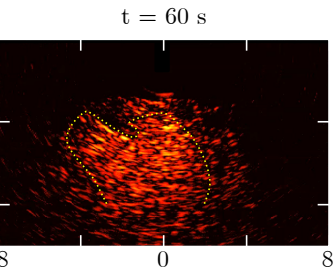

0

Fig. 3. All-optical ultrasound imaging of inhomogeneous tissue. (a) Photograph of the inhomogeneous tissue sample (pork belly) post-ablation $(t>60 \mathrm{~s})$, showing a cross-section coinciding with the 2D all-optical ultrasound image plane. The ablation lesion (the white, "cloudy" region indicated by the red arrow head) is clearly visible. (b) Schematic of the image plane (ablation lesion not shown). A section of pork belly was positioned a distance of 3 mm away from the imaging aperture (dashed line), and placed in direct contact with the tip of the ablation catheter that was positioned behind the tissue. A single fibre-optic detector was placed directly within the image plane, centered laterally, and axially offset by $1.0 \mathrm{~mm}$. (c) All-optical ultrasound images of the tissue sample at different time points during the treatment. Time $t=0$ corresponds to the start of the $60 \mathrm{~s}-l o n g$ ablation. The dashed curves (left panel) show the location of the tissue and catheter tip; the yellow dotted curves (middle and right panels) the approximate location and shape of the ablation lesion. The full video of this experiment is available on youtu.be/6ZD7cEj4AYs.

[9] J Rebling, FJO Landa, XL Den-Ben, A Douplik, and D Razansky. Integrated catheter for simultaneous radio frequency ablation and optoacoustic monitoring of lesion progression. Optics letters, 43(8):18861889, 2018.

[10] E Vannacci, L Belsito, F Mancarella, M Ferri, GP Veronese, A Roncaglia, and E Biagi. Miniaturized fiber-optic ultrasound probes for endoscopic tissue analysis by micro-opto-mechanical technology. Biomedical microdevices, 16(3):415-426, 2014.

[11] RJ Colchester, EZ Zhang, CA Mosse, PC Beard, I Papakonstantinou, and AE Desjardins. Broadband miniature optical ultrasound probe for high resolution vascular tissue imaging. Biomedical Optics Express, 6(4):1502-1511, 2015.

[12] E Vannacci, S Granchi, L Belsito, A Roncaglia, and E Biagi. Wide bandwidth fiber-optic ultrasound probe in moms technology: Preliminary signal processing results. Ultrasonics, 75:164-173, 2017.

[13] MC Finlay, CA Mosse, RJ Colchester, S Noimark, EZ Zhang, S Ourselin, PC Beard, RJ Schilling, IP Parkin, I Papakonstantinou, and AE Desjardins. Through-needle all-optical ultrasound imaging in vivo: a preclinical swine study. Light: Science \& Applications, 6(12):e17103, 2017.

[14] EJ Alles, S Noimark, E Maneas, EZ Zhang, IP Parkin, PC Beard, and AE Desjardins. Video-rate all-optical ultrasound imaging. Biomedical Optics Express, 9(8):3481-3494, 2018.

[15] PC Beard. Biomedical photoacoustic imaging. Interface Focus, 1(4):602-631, 2011.

[16] S Noimark, RJ Colchester, BJ Blackburn, EZ Zhang, EJ Alles, S Ourselin, PC Beard, I Papakonstantinou, IP Parkin, and AE Desjardins. Carbon-nanotube-PDMS composite coatings on optical fibres for alloptical ultrasound imaging. Advanced Functional Materials, 26(35), 2016.

[17] S-L Chen. Review of laser-generated ultrasound transmitters and their applications to all-optical ultrasound transducers and imaging. Applied Sciences, 7(1):25, 2016.

[18] S Noimark, RJ Colchester, RK Poduval, E Maneas, EJ Alles, T Zhao, EZ Zhang, M Ashworth, E Tsolaki, and AH Chester. Polydimethylsiloxane composites for optical ultrasound generation and multimodality imaging. Advanced Functional Materials, 28(9), 2018.

[19] EZ Zhang and PC Beard. Characteristics of optimized fibre-optic ultrasound receivers for minimally invasive photoacoustic detection. In Proceedings SPIE BiOS, pages 932311-932311-9. International Society for Optics and Photonics, 2015.

[20] E Zhang, JG Laufer, and PC Beard. Backward-mode multiwavelength photoacoustic scanner using a planar Fabry-Pérot polymer film ultrasound sensor for high-resolution three-dimensional imaging of biological tissues. Applied Optics, 47(4):561-577, 2008.

[21] JA Guggenheim, J Li, TJ Allen, RJ Colchester, S Noimark, OO Ogunlade, IP Parkin, I Papakonstantinou, AE Desjardins, EZ Zhang, and PC Beard. Ultrasensitive plano-concave optical microresonators for ultrasound sensing. Nature Photonics, 11(11):714-719, 2017.

[22] M Karaman, P-C Li, and M O'Donnell. Synthetic aperture imaging for small scale systems. Ultrasonics, Ferroelectrics, and Frequency Control, IEEE Transactions on, 42(3):429-442, 1995.

[23] EJ Patterson, CH Scudamore, DA Owen, AG Nagy, and AK Buczkowski. Radiofrequency ablation of porcine liver in vivo: effects of blood flow and treatment time on lesion size. Annals of surgery, 227(4):559, 1998.

[24] HW Huang. Influence of blood vessel on the thermal lesion formation during radiofrequency ablation for liver tumors. Medical physics, 40(7), 2013. 